\title{
Construction of recombinant lentivirus vector for tumor vasoinhibitory peptide alphastatin gene delivery
}

\author{
S.W. GUO*, H.M. CHE* and W.Z.LI \\ Department of Neurosurgery, First Affiliated Hospital, Medical School of Xi'an Jiaotong University, Shaanxi 710061, P.R. China
}

Received May 27, 2010; Accepted July 26, 2010

DOI: $10.3892 / \mathrm{mmr} .2010 .350$

\begin{abstract}
Angiogenesis is a prerequisite for tumor progression and metastasis. Alphastatin, as an endogenous angiogenesis inhibitor, was recently used as an anticancer agent in several tumor models. We constructed recombinant self-inactivating lentivirus vectors expressing alphastatin and evaluated their ability to transfer genes into human umbilical vein endothelial cells (HUVECs) as well as their antiangiogenic activities in vitro. Recombinant self-inactivating lentiviral vectors efficiently and stably transduced endothelial cells, and lentivirus-transduced HUVECs were capable of sustainedly secreting the antiangiogenesis peptide alphastatin. Long-term expression and secretion of alphastatin resulted in significant inhibition of endothelial cell angiogenesis induced by vascular endothelial growth factor. This report presents the first use of lentivirus-based vectors to deliver the endogenous angiogenesis inhibitor alphastatin, and suggests the potential utility of antiangiogenic gene therapy with lentiviral vectors for the treatment of cancer.
\end{abstract}

\section{Introduction}

It is widely recognized that angiogenesis is a critical process required by solid tumors to support their growth. Within a given microenvironment, the angiogenic response results from a balance between proangiogenic and antiangiogenic factors, secreted by tumor cells and components of the stroma, which result in the activation of angiogenesis followed by tumor outgrowth (1). Several studies have demonstrated that endogenous angiogenesis inhibitors in the body, including angiostatin (2), endostatin (3) and alphastatin (4), contribute to the suppression of pathologic angiogenesis.

Alphastatin, a 24-amino acid peptide derived from the amino terminus of the $\alpha$-chain of human fibrinogen, has been shown to have potent antiangiogenic properties in vitro and in vivo (4-6). However, peptide-based therapeutic strategies

Correspondence to: Professor S.W. Guo, Department of Neurosurgery, the First Affiliated Hospital, Medical School of Xi'an Jiaotong University, Xi'an, Shaanxi 710061, P.R. China

E-mail: gsw1962@126.com

${ }^{*}$ Contributed equally

Key words: lentivirus, vector, alphastatin, antiangiogenesis using these antiangiogenic factors have been limited by significant difficulties including large-scale protein production, a short circulating half-life and low peptide stability in vivo, and the requirement of long-term administration to maintain tumor suppression.

Delivery of the genes encoding angiogenic inhibitors using viral vectors represents a more effective strategy that has the potential for in situ production of higher local concentrations of these inhibitors than those achieved by systemic infusion of antiangiogenic peptides. This will more efficiently prevent the induction of new blood vessel formation, and will minimize potential systemic side effects (7-9). Therefore, an efficient gene delivery system that achieves stable long-term expression of the peptides is necessary.

Third generation lentiviruses represent an attractive new vector system that allows permanent integration of the delivered transgene and definitively increases the level of biosafety (10). There are no previous reports of lentivirus-based vectors expressing the angiogenesis inhibitor alphastatin. Here, we successfully constructed recombinant lentivirus vectors capable of transducing endothelial cells for long-term expression of alphastatin, which significantly inhibited angiogenesis in vitro.

\section{Materials and methods}

Reagents. Endothelial cell growth supplement (ECGS), Dulbecco's modified Eagle's medium (DMEM), 3-(4,5dimethylthiazol-2-yl)-2,5-diphenyltetrazolium bromide (MTT) and dimethyl-sulfoxide (DMSO) were purchased from Sigma-Aldrich. Growth factor-reduced (GFR) Matrigel was purchased from Becton Dickinson. Recombinant human vascular endothelial growth factor (VEGF) was acquired from PeproTech. Fetal bovine serum (FBS) was purchased from Gibco (USA). Restriction enzyme BamHI and EcoRI were purchased from New England Biolabs.

Cells and cell culture. Freshly delivered umbilical cords were obtained from natural births, and human umbilical vein endothelial cells (HUVECs) were isolated from human umbilical cord veins via collagenase digestion as previously described (11). The cells were cultured in EGM-2 supplemented with $20 \% \mathrm{FBS}$ at $37^{\circ} \mathrm{C}$ in a humidified atmosphere of $5 \% \mathrm{CO}_{2}$. Confluent cultures between the third and eighth passages were employed in the functional assays. 
Generation of expression vector. A prokaryotic expression plasmid vector pBV220/NT4-Al encoding the NT4-Al (Neurotrophin-4 signal peptide, pro-region sequences and alphastatin) fusion gene fragment was constructed as previously described (12-15). After digestion with restriction enzymes, the resulting NT4-Al gene with BamHI and EcoR I sites from the pBV220/NT4-Al plasmid was inserted into the multiple cloning site of the expression plasmid pWPXL/ GFP-IRES-GFP (a kind gift from the University of California, San Diego, CA, USA) containing a multiple cloning site and the gene encoding green fluorescent protein (GFP). The resultant vector plasmid pWPXL/NT4-Al-IRES-GFP was digested with restriction enzyme to test whether the NT4-Al cDNA fragment was successfully inserted into the lentivirus shuttle plasmid (Fig. 1A).

Production and titration of recombinant lentivirus vectors. An improved third-generation lentivirus system has been developed $(10,16)$. This system comprises the following plasmids: pMDLG/pRRE and pRSV/REV, packaging plasmids in which all accessory genes (vif, vpr, vpu and nef) and regulatory genes (tat and rev) are deleted; pMD2.G, an envelope plasmid for vesicular stomatitis virus $\mathrm{G}$ glycoprotein and Rev protein; and the SIN plasmid pWPXL/GFP-IRES-GFP, which contains a multiple cloning site and the gene encoding GFP. The recombinant lentivirus vector of NT4-Al was generated by cotransfection of $293 \mathrm{~T}$ cells with $20 \mu \mathrm{g}$ pWPXL/NT4-Al, $12 \mu \mathrm{g}$ pWPXL/NT4-Al-IRES-GFP, $10 \mu \mathrm{g}$ pRSV/REV and $10 \mu \mathrm{g}$ pMD2.G plasmids in $10-\mathrm{cm}$ dishes with Lipofectamine 2000 (Invitrogen, USA). 293T cells were cultured in DMEM containing $10 \%$ heat-inactivated FBS. Culture supernatants were collected every $24 \mathrm{~h}$ for 3 days, filtered through a $0.45-\mu \mathrm{m}$ pore size filter and concentrated two times by ultracentrifugation at $50,000 \mathrm{x} \mathrm{g}$ at $20^{\circ} \mathrm{C}$ for $120 \mathrm{~min}$. The viral supernatants were concentrated 1,000 times by ultracentrifugation, and finally resuspended in sterile phosphate-buffered saline (PBS) and stored at $-80^{\circ} \mathrm{C}$ until use. The virus titers were determined on $293 \mathrm{~T}$ cells by measuring GFP expression using flow cytometry.

Infection of HUVECs with lentivirus vectors. On the day of infection, cells were plated at a density of $4 \times 10^{4}$ cells/well in 96-well plates along with lenti-NT4-Al or lenti-GFP at different multiplicities of infection (MOI) in serum-free growth medium containing $5 \mu \mathrm{g} / \mathrm{ml}$ polybrene. Serum-containing growth medium was added after $4 \mathrm{~h}$, and then replaced after $48 \mathrm{~h}$. Reporter gene expression was examined using fluorescent microscopy on days 4 and 5 post-infection. HUVEC-NT4Al-GFP and HUVEC-GFP cells were then passaged and prepared for subsequent assays.

Alphastatin secretion expression from transduced cells. Following gene transfer into HUVECs, the secretion expression levels of the alphastatin protein were assessed by SDS-PAGE and mass spectrometric analysis. Briefly, HUVEC-NT4-Al-GFP or HUVEC-GFP cells were incubated for $48 \mathrm{~h}$ in serum-free medium. Each sample (20 $\mu \mathrm{l})$ was run on SDS-PAGE gels and then transferred onto nitrocellulose membranes (Pall Gelman Sciences, Ann Arbor, MI, USA). The gels were then stained with Coomassie Brilliant Blue to determine the secretion protein and its purity in the cell culture supernatant. The molecular weights of the samples were estimated using mass spectrometry analysis to confirm that alphastatin had been secreted into the cell culture supernatant.

Cell culture supernatant collection. Cell culture supernatants were collected from the culture media of HUVEC-GFP (HU-Null) and HUVEC-NT4-Al-GFP (HU-Al) cells. Briefly, HU-Null or HU-Al cells were cultured until sub-confluent in DMEM media containing $100 \mathrm{~g} / \mathrm{ml}$ ECGS for $24 \mathrm{~h}$. The medium was then replaced with DMEM containing $1 \%$ FBS for a further $24 \mathrm{~h}$. The conditioned medium was collected and concentrated 40-fold through a Microcon-10 column (Millipore, Bedford, MA, USA), then stored at $-80^{\circ} \mathrm{C}$ for subsequent assays.

Effect of lentivirus vector transfection on HUVEC cell growth. The MTT assay was used to assess the proliferation of the HUVECs (17). Parantal HUVECs, HUVEC-GFP (HU-Null) and HUVEC-NT4-Al (HU-Al), were individually prepared and seeded into 96 -well plates at $3 \times 10^{4}$ cells $/ \mathrm{ml}$ for 24,48 and $72 \mathrm{~h}$. At these time points, a quarter volume of MTT solution (2 $\mathrm{mg}$ MTT/ml PBS) was added to each well and each plate was incubated for $4 \mathrm{~h}$ at $37^{\circ} \mathrm{C}$, resulting in an insoluble purple formazan product. The medium was aspirated and the precipitates were dissolved in $150 \mu \mathrm{l}$ of DMSO buffered at $\mathrm{pH}$ 10.5. The absorbance was then read at $490 \mathrm{~nm}$ using a Dynex enzyme-linked immunosorbent assay (ELISA) plate reader (Ashford, Middlesex, UK).

Cell migration assay. The cell migration assay was adapted from Malinda et al (18) and involved the use of a 24-well microchemotaxis chamber (Neuro Probe, AM) with $8-\mu \mathrm{m}$ pore size polycarbonate membranes (Neuro Probe, AM), the under surfaces of which were coated with $10 \mu \mathrm{l}$ of GFR Matrigel. HUVEC-Null, HUVEC-Al or HUVECs were grown to sub-confluence in the lower chambers in DMEM containing $100 \mathrm{~g} / \mathrm{ml}$ ECGS for $24 \mathrm{~h}$. The medium was then replaced with DMEM containing $1 \%$ FBS for a further $24 \mathrm{~h}$. VEGF, either alone $(10 \mathrm{ng} / \mathrm{ml})$ or combined with cell conditioned culture supernatant $(25 \mu \mathrm{l} / \mathrm{ml})$, was added to the lower chambers and incubated for $48 \mathrm{~h}$. The HUVEC cell suspension ( $1 \times 10^{5}$ cells/ $\mathrm{ml}$ ) was then added to the upper chambers and incubated at $37^{\circ} \mathrm{C}$ for $8 \mathrm{~h}$. Migrated cells adhering to the under surface of the membranes were fixed with $4 \%$ paraformaldehyde and stained with H\&E. The migrated cells were then counted under an optical microscope at a magnification of $\mathrm{x} 20$.

Tube formation assay. For the tube formation assay, 24-well plates were coated with GFR Matrigel (300 $\mu \mathrm{l} /$ well). Endothelial cells seeded onto this matrix usually migrated and formed tubules within $6 \mathrm{~h}$ of plating. HUVECs were seeded at $2 \times 10^{5}$ cells $/ \mathrm{ml}$ and incubated for $6 \mathrm{~h}$ in $500 \mu \mathrm{l}$ $\mathrm{DMEM}+1 \%$ FBS (control), or in DMEM $+25 \mu \mathrm{l} / \mathrm{ml}$ cell culture supernatant, with or without $20 \mathrm{ng} / \mathrm{ml}$ VEGF. After 8 $\mathrm{h}$, the endothelial cell-derived tube-like structures were visualized using an inverted microscope and photographed at a magnification of x20. Tube-like structure formation was also quantified by calculating the tube areas. 
A

Transfer vector (pWPXL-Alphastatin)

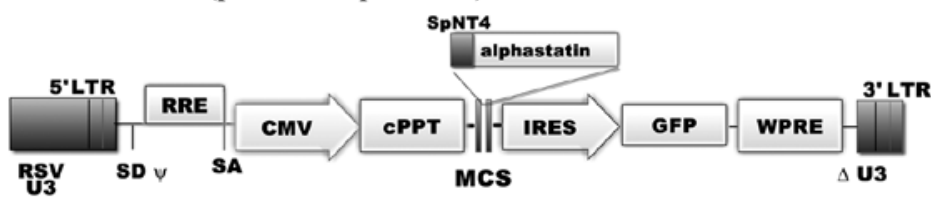

Packing construct (pMDlg-pRRE pRsv-RRE)

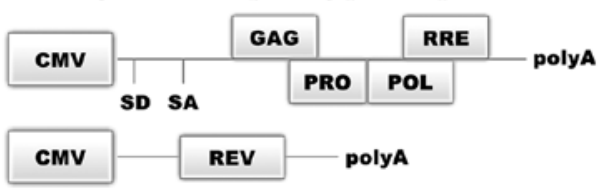

Env-coding plasmid (pMD2.G)

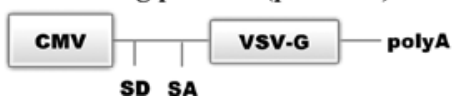

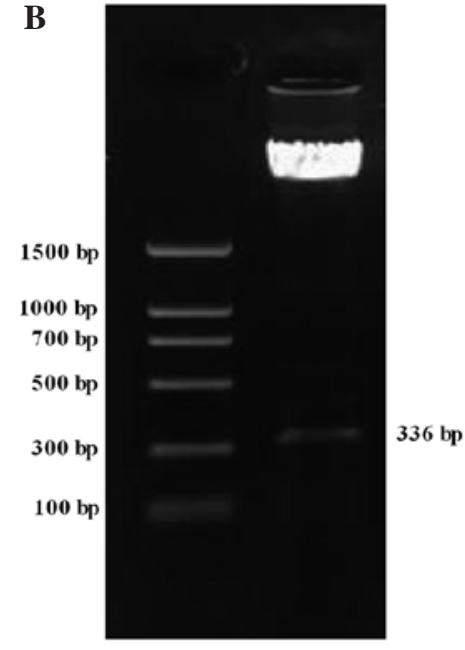

Figure 1. Packaging system of Lent-NT4-Al-GFP and enzyme digestion identification of pWPXL-NT4-Al. (A) Self-inactivating lentivirus vectors: the human neurotrophin-4 signal peptide and pro-region fusion sequences (SpNT4) were cloned into lentiviral transfer vector plasmid pWPXL/GFP to construct pWPXL-NT4-A1. Recombinant lentivirus vectors were produced by co-transfection of the envelope plasmid pMD2.G, packaging plasmid pMDlg-RRE and pRsv-RRE, and transfer vector plasmid. LTR, long terminal repeat; RSV U3, U3 region from Rous sarcoma virus; $\psi$, packaging signal; SD, splice donor; SA, splice acceptor; RRE, Rev response element; CMV, cytomegalovirus promoter; MCS, multiple cloning site; IRES, internal ribosome entry site; GFP, green fluorescent protein marker gene; $\triangle \mathrm{U} 3$, self-inactivating deletion in $\mathrm{U} 3$ region; poly A, polyadenylation signal; VSVG, vesicular stomatitis virus $\mathrm{G}$ protein envelope. (B) The positive clone pWPXL/NT4-Al was digested with restriction enzyme BamHI and EcoRI. Quantitative competitive PCR revealed the cDNA fragment to be $336 \mathrm{bp}$.

Statistical analysis. Data are expressed as the mean \pm SEM, and representative data from one of three replicate experiments are shown. Differences between the groups were determined using one-way ANOVA followed by the Student's t-test. A P-value of $<0.05$ was considered significant.

\section{Results}

Construction and identification of expression plasmid and production of recombined lentivirus vectors. The lentivirus expression plasmid we constructed contained the elements required for virion packaging, such as $5^{\prime}$ and $3^{\prime}$ long terminal repeats and a $\psi$ packaging signal. The packaging system of Lent-NT4-Al-GFP is shown in Fig. 1A. The DNA sequence of the NT4-Al fusion gene was identical to that of NT4 and the 24 amino acid alphastatin. The length of NT4-Al was 336 bp, including a 247-bp fragment of a human neurotrophin-4 signal peptide sequence and a pro-region with two restriction sites (BamHI and EcoRI). To test whether the NT4-alphastatin sequences were cloned into the plasmid, enzyme digestion identification and PCR analysis were performed. Fairly steady 336-bp sequences were achieved and the recombinant expression plasmid pWPXL/NT4-Al was shown to be correctly constructed (Fig. 1B). After co-infection by four plasmids of 293 T cells, lentivirus vectors were produced and flow cytometry analysis revealed that the virus titers were $3.4 \times 10^{8} \mathrm{TU} / \mathrm{ml}$. pWPXL/GFP, a vector containing only the IRES-GFP expression cassette without any transgene expression upstream of the IRES, was used as a negative control.

Production and secretion of antiangiogenic peptides from lentivirus vectors. GFP expression was detected in HUVEC cells at $72 \mathrm{~h}$ post-infection by fluorescent microscopic analysis (Fig. 2A). Typically, at a MOI of $40,>98 \%$ of the transduced cells were found to be GFP-positive using fluorescence-activated cell sorting analysis. Secretion protein molecular weight was identified using mass spectrometry analysis. The data revealed that experimental protein molecular weight was equal to the theoretical value of alphastatin (Fig. 2B). Production of secretory forms of human alphastatin after gene transfer by the vesicular stomatitis virus G protein (VSV-G)-pseudotyped SIN lentivirus vector was confirmed by SDS-PAGE. Analysis revealed that the relative molecular weight of the expressed product was 2.3-2.5 kDa, consistent with the expected value (Fig. 2C).

Effects of alphastatin transduction on endothelial cell growth and proliferation. To evaluate the effects of alphastatin transduction and expression on the growth of HUVECs in vitro, the relative growth rates of $\mathrm{HU}-\mathrm{Null}, \mathrm{HU}-\mathrm{Al}$ and parental HUVECs were compared using the MTT assay. As shown in Fig. 3A, there was no significant difference between the growth rates of HU-Null, HU-Al and parental HUVECs, suggesting that neither the lentivirus transduction procedure nor the overexpression of GFP or alphastatin affected the intrinsic rate of cellular proliferation in these cells.

\section{Effects of alphastatin on VEGF-induced HUVEC migration} and tube formation. Experiments were performed to determine whether alphastatin affected the three main stages of angiogenesis - endothelial cell proliferation, migration and tubule formation - induced by VEGF in vitro. Exposure to alphastatin-containing culture supernatant significantly inhibited VEGF-induced migration in a Boyden chamber assay ( $\mathrm{P}<0.001$; Fig. 3B). In addition, tube formation by HUVECs was significantly inhibited by alphastatin-containing culture supernatant $(25 \mu \mathrm{l} / \mathrm{ml} ; \mathrm{P}<0.004)$ in the presence of $20 \mathrm{ng} /$ ml VEGF (Fig. 4). However, HUVEC proliferation was not affected by alphastatin-containing culture supernatant. 
A
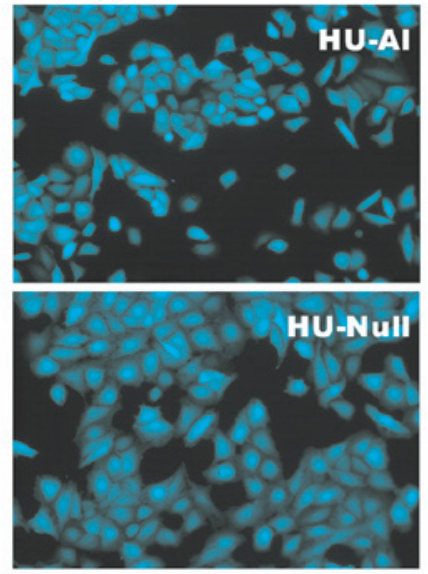

C

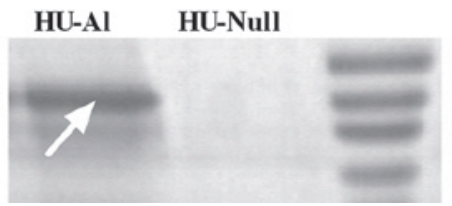

$3.4 \mathrm{kDa}$

$2.3 \mathrm{kDa}$

$1.7 \mathrm{kDa}$
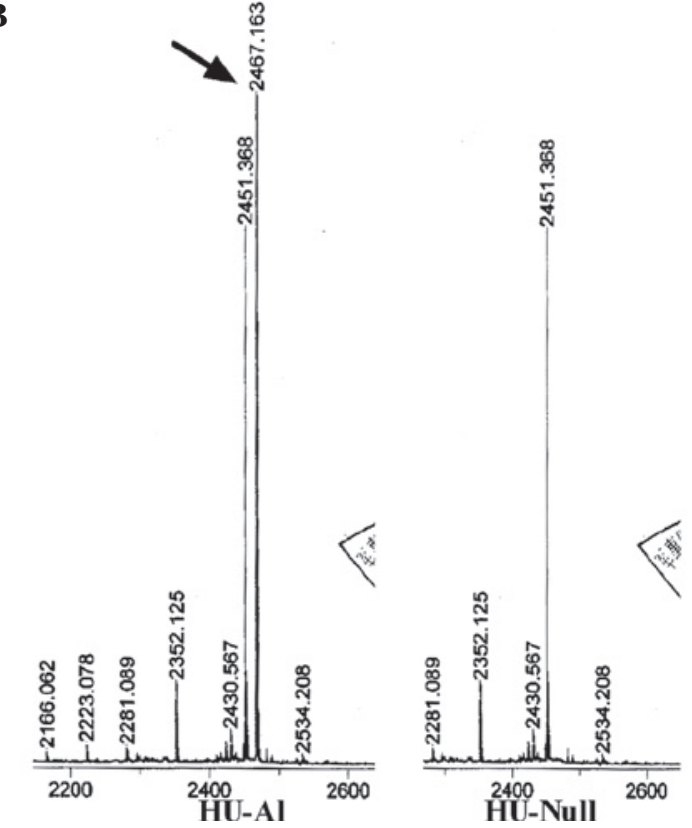

Figure 2. Transduction of HUVECs and identification of secretion protein. (A) HUVECs infected with Lent-NT4-Al-GFP or Lent-GFP showed both nuclear and cytoplasmic expression of GFP. (B) The difference between the two types of cell culture supernatants was that only HU-Al supernatant had protein with a molecular weight of $2,467.163 \mathrm{kDa}$ (equal to the theoretical value of alphastatin, indicated by the black arrow). (C) SDS-PAGE determined that only HU-Al cells expressed the protein alphastatin, and that this production had a higher purity (indicated by the white arrow).

A

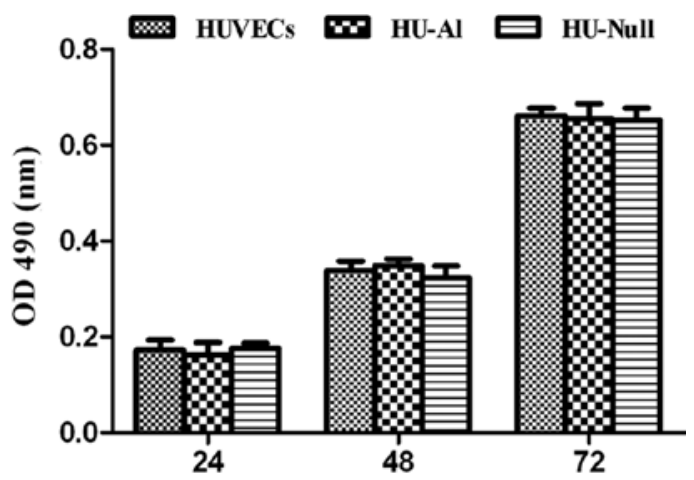

B

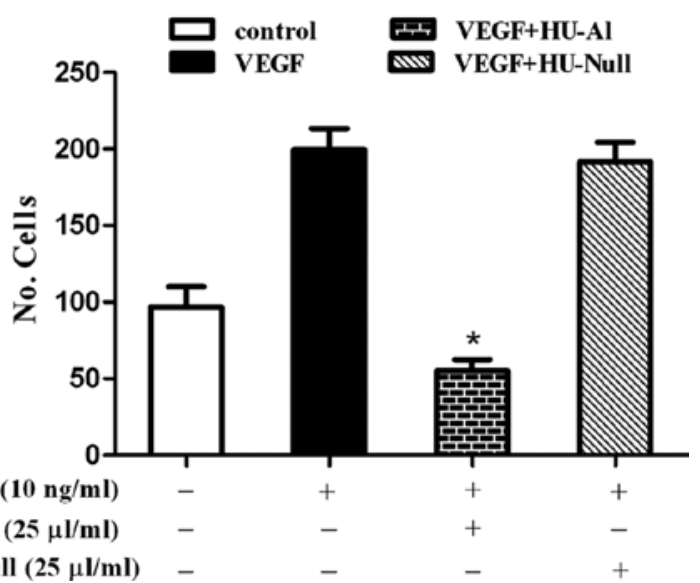

Figure 3. The effects of alphastatin transduction on endothelial cell growth and proliferation and of alphastatin on HUVEC migration. (A) Parental HUVECs, HU-Al and HU-Null cell proliferation over a 72-h period. The data revealed that the parental HUVECs and cells after infection with lent-NT4-Al and lent-GFP had equal proliferation rates. (B) HUVEC migration across a collagen-coated filter in response to medium alone (control) or medium containing $10 \mathrm{ng} / \mathrm{ml}$ VEGF. Alphastatin significantly inhibited VEGF-induced HUVEC migration. The results are expressed as the mean \pm SEM. ${ }^{*} \mathrm{P}<0.0001$ for VEGF + HU-Al vs. VEGF.

\section{Discussion}

Lentivirus vectors are capable of permanently integrating into the target cells. In the present study, recombinant lentivirus vectors expressing secretory forms of alphastatin were constructed and tested for their antiangiogenic activities. These lentivirus vectors efficiently infected endothelial cells, resulting in the expression and secretion of alphastatin at levels sufficient to achieve significant inhibition of endothelial cell angiogenesis. To our knowledge, this is the first report of lentivirus-based vectors encoding these antiangiogenic factors.

Lentiviral vectors integrate their cDNA into both dividing and non-dividing cells (19). A third-generation SIN lentivirus vector, in which the U3 region of the 3' LTR (including the TATA box) was deleted, thus abolishing any LTR promoter activity (10), enhanced the security level of transgene expression significantly. In addition, VSV-G pseudotyped lentivirus vectors, which bind to cell surface phospholipids (20), have a potential advantage in achieving efficient gene delivery to endothelial cells. In this study, using a third-generation lentivirus, we successfully constructed recombinant lentivirus vectors of the NT4-Al fusion gene. In this delivery system, the alphastatin sequence alone is not sufficient for efficient translation and secretion in mammalian cells (21-24); therefore, the human neurotrophin- 4 signal peptide and pro-region sequence (NT4) were fused in-frame to the end of the coding sequence and a stop codon was appended to the end of the alphastatin coding sequence (Fig. 1A). In this way, the signal peptide and pro-region of human neurotrophin- 4 were able to contribute to alphastatin sufficient secretion expression in mammalian cells $(14,15,25)$.

Endothelial cells were selected as the target transduction cells in this study. As tumor growth is highly dependent on angiogenesis, particularly in brain malignant gliomas 


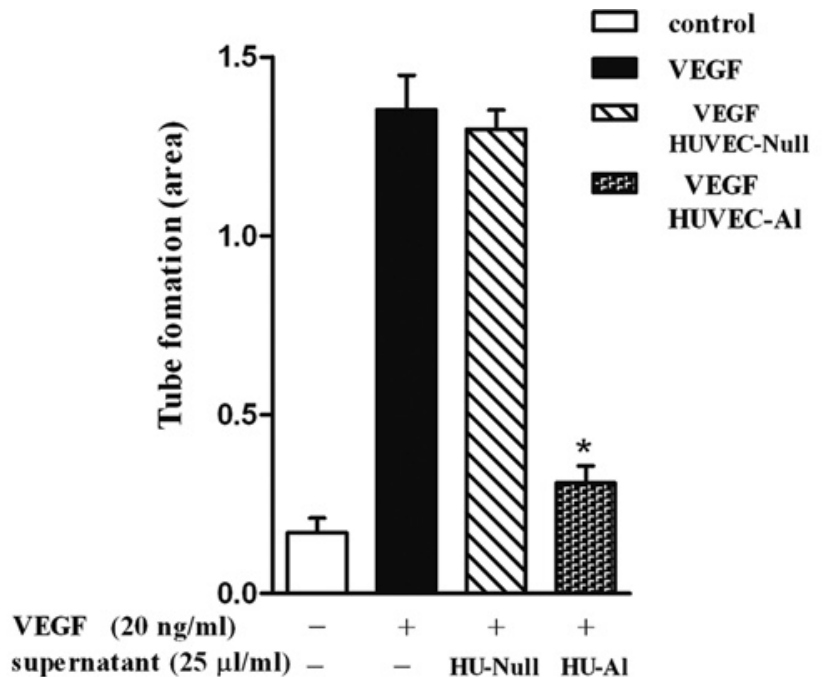

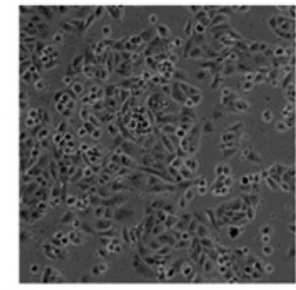

control

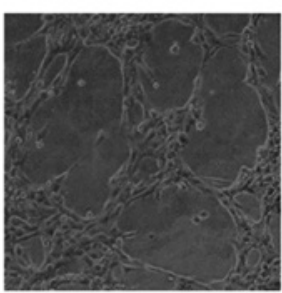

VEGF HUVEC-Null

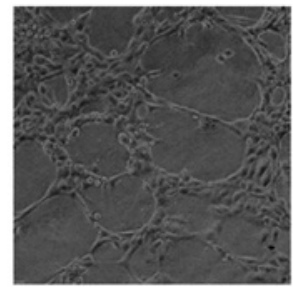

VEGF

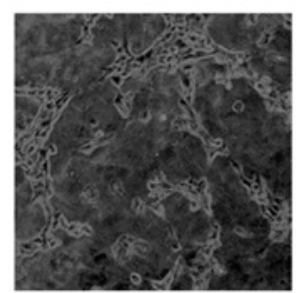

VEGF HUVEC-AI

Figure 4. The effect of alphastatin on tubule formation induced by VEGF. Tubule formation by HUVECs exposed to culture supernatants from HU-Al, HU-Null cells, in the presence or absence of VEGF (20 ng/ml). The HU-Al supernatant significant inhibited HUVECs tubule formation induced by VEGF. The data are expressed as mean \pm SEM. ${ }^{*} \mathrm{P}<0.004$, VEGF HU-Al vs. VEGF.

$(26,27)$, the introduction of a vector into endothelial cells is, by contrast, exponential or geometric (28). In fact, with intravenous administration, viral vectors easily access the target cells without having to cross through the vessel wall to reach the targets. Additionally, in construction co-implanting tumor models (infected HUVECs and tumor cells; data not shown) sustained secretory protein alphastatin from HUVECs stimulated an intravenous route of administration, which was capable of avoiding repeat direct administration with expensive recombined lentivirus. Thus, introducing HUVECs as target transduction cells practically represented a systemic antiangiogenesis therapeutic strategy. However, the effect of endothelial cells promoting tumor growth should be considered (29). This increased the practical application of the alphastatin gene delivery therapeutic strategy.

Furthermore, alphastatin is a potent inhibitor of activated endothelial cells in vitro and in vivo since, as an agent that specifically targets activated endothelium in areas of angiogenesis rather than endothelial cells in the quiescent vasculature, particularly in tumor neovascularization, the receptors for alphastatin may be expressed only on endothelium activated by VEGF or bFGF in tumor vessels. Here, we determined that the cell culture supernatant of infected HU-Al significantly inhibited VEGF-induced endothelial migration and tube formation, but not proliferation in the initial stage of angiogenesis. In addition, our data showed that HU-Al cells transfected by recombined lentivirus and the control HUVECs demonstrated equal growth rates, and alphastatin inhibited VEGF-induced HUVECs, but not normal HUVECs. This suggests that transduced endothelial cells were capable of sustainedly secreting alphastatin, which markedly suppressed the initial stage of angiogenesis (1). Moreover, lentiviral infection and alphastatin transduction did not alter the growth characteristics of HUVEC cells or affect quiescent endothielial cells. Therefore, the secretion expression protein alphastatin from endothelial cells inhibited angiogenesis in an autocrine fashion when endothelial cells were activated by VEGF, or in a paracrine fashion when quiescent endothelial cells were presented.
In conclusion, we demonstrated the use of recombinant lentivirus vectors for the stable transduction of HUVEC cells to achieve the secretion of alphastatin for the local suppression of angiogenesis. This could serve as an endogenous source of constitutively secreted antiangiogenic factors, an approach that may have potential in the prevention of distant metastases and local recurrences of malignancies after surgical removal of the primary tumor.

\section{Acknowledgements}

This study was supported by a grant from the National Natural Science Foundation of China (No. 30672162).

\section{References}

1. Hanahan D and Folkman J: Patterns and emerging mechanisms of the angiogenic switch during tumorigenesis. Cell 86: 353-364, 1996.

2. O'Reilly MS, Holmgren L, Shing Y, et al: Angiostatin: a novel angiogenesis inhibitor that mediates the suppression of metastases by a Lewis lung carcinoma. Cell 79: 315-328, 1994.

3. O'Reilly MS, Boehm T, Shing Y, et al: Endostatin: an endogenous inhibitor of angiogenesis and tumor growth. Cell 88: 277-285, 1997.

4. Staton CA, Brown NJ, Rodgers GR, et al: Alphastatin, a 24-amino acid fragment of human fibrinogen, is a potent new inhibitor of activated endothelial cells in vitro and in vivo. Blood 103: 601-606, 2004.

5. Staton CA, Stribbling SM, Garcia-Echeverria C, et al: Identification of key residues involved in mediating the in vivo anti-tumor/anti-endothelial activity of Alphastatin. J Thromb Haemost 5: 846-854, 2007.

6. Chen L, Li T, Li R, Wei B and Peng Z: Alphastatin downregulates vascular endothelial cells sphingosine kinase activity and suppresses tumor growth in nude mice bearing human gastric cancer xenografts. World J Gastroenterol 12: 4130-4136, 2006.

7. Shichinohe T, Bochner BH, Mizutani K, et al: Development of lentiviral vectors for antiangiogenic gene delivery. Cancer Gene Ther 8: 879-889, 2001.

8. Folkman J: Antiangiogenic gene therapy. Proc Natl Acad Sci USA 95: 9064-9066, 1998.

9. Kong HL and Crystal RG: Gene therapy strategies for tumor antiangiogenesis. J Natl Cancer Inst 90: 273-286, 1998.

10. Zufferey R, Dull T, Mandel RJ, et al: Self-inactivating lentivirus vector for safe and efficient in vivo gene delivery. J Virol 72: 9873-9880, 1998. 
11. Jaffe EA, Nachman RL, Becker CG and Minick CR: Culture of human endothelial cells derived from umbilical veins. Identification by morphologic and immunologic criteria. J Clin Invest 52: 2745-2756, 1973 .

12. Guo SW, Che HM, Li WZ, Yang GX and Lian MX: Construction of prokaryotic expression vector for NT4-Al fusion gene. Chin J Mini Invas Neurosurg 14: 319-321, 2009.

13. Guo SW, Yang Y, Wang QY, Lian MX, Li T and Wang DF: Preparation of the antiangiogenic peptide alphastatin recombinant adeno-associated virus. Chin J Neurosurg Dis Res 5 518-521, 2006

14. Li Y, Qiu S, Song L, Yan Q and Yang G: Secretory expression of p53(N15)-Ant following lentivirus-mediated gene transfer induces cell death in human cancer cells. Cancer Invest 26: 28-34, 2008.

15. Xiaojiang T, Jinsong Z, Jiansheng W, Chengen $P$, Guangxiao $Y$ and Quanying W: Adeno-associated virus harboring fusion gene NT4-ant-shepherdin induce cell death in human lung cancer cells. Cancer Invest 28: 465-471, 2010.

16. Miyoshi H, Blomer U, Takahashi M, Gage FH and Verma IM Development of a self-inactivating lentivirus vector. J Virol 72 : 8150-8157, 1998.

17. Liu J, Kolath J, Anderson J, et al: Positive interaction between 5-FU and FdUMP[10] in the inhibition of human colorectal tumor cell proliferation. Antisense Nucleic Acid Drug Dev 9 481-486, 1999.

18. Malinda KM, Ponce L, Kleinman HK, Shackelton LM and Millis AJ: Gp38k, a protein synthesized by vascular smooth muscle cells, stimulates directional migration of human umbilical vein endothelial cells. Exp Cell Res 250: 168-173, 1999.
19. Naldini L, Blomer U, Gallay P, et al: In vivo gene delivery and stable transduction of nondividing cells by a lentiviral vector. Science 272: 263-267, 1996.

20. Schlegel R, Tralka TS, Willingham MC and Pastan I: Inhibition of VSV binding and infectivity by phosphatidylserine: is phosphatidylserine a VSV-binding site? Cell 32: 639-646, 1983.

21. Zanen G, Antelmann H, Meima R, et al: Proteomic dissection of potential signal recognition particle dependence in protein secretion by Bacillus subtilis. Proteomics 6: 3636-3648, 2006.

22. Nagai K, Oubridge C, Kuglstatter A, Menichelli E, Isel C and Jovine L: Structure, function and evolution of the signal recognition particle. EMBO J 22: 3479-3485, 2003.

23. Muller JP, Bron S, Venema G and van Dijl JM: Chaperone-like activities of the CsaA protein of Bacillus subtilis. Microbiology 146: 77-88, 2000 .

24. Tjalsma H, Antelmann H, Jongbloed JD, et al: Proteomics of protein secretion by Bacillus subtilis: separating the 'secrets' of the secretome. Microbiol Mol Biol Rev 68: 207-233, 2004

25. Fan G, Egles C, Sun Y, et al: Knocking the NT4 gene into the BDNF locus rescues BDNF deficient mice and reveals distinct NT4 and BDNF activities. Nat Neurosci 3: 350-357, 2000.

26. Louis DN, Pomeroy SL and Cairncross JG: Focus on central nervous system neoplasia. Cancer Cell 1: 125-128, 2002.

27. Papale A, Cerovic M and Brambilla R: Viral vector approaches to modify gene expression in the brain. J Neurosci Methods 185 1-14, 2009.

28. Liu Y and Deisseroth A: Tumor vascular targeting therapy with viral vectors. Blood 107: 3027-3033, 2006.

29. Calabrese C, Poppleton H, Kocak M, et al: A perivascular niche for brain tumor stem cells. Cancer Cell 11: 69-82, 2007. 